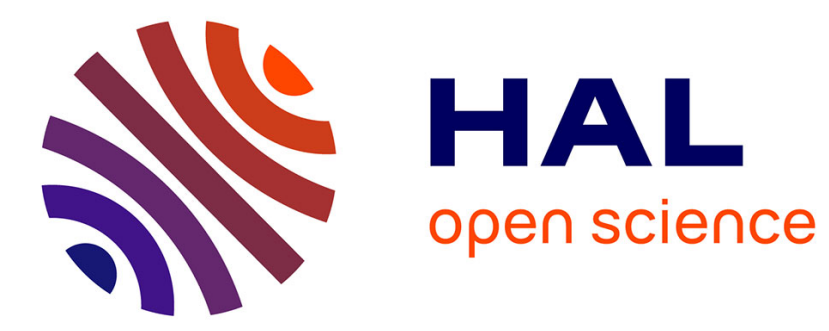

\title{
Polarimetric Analysis Using the Algebraic Real Representation of the Scattering Matrix
}

Madalina Ciuca, Gabriel Vasile, Michel Gay, Andrei Anghel, Silviu Ciochina

\section{To cite this version:}

Madalina Ciuca, Gabriel Vasile, Michel Gay, Andrei Anghel, Silviu Ciochina. Polarimetric Analysis Using the Algebraic Real Representation of the Scattering Matrix. IGARSS 2021 - 2021 IEEE International Geoscience and Remote Sensing Symposium, Jul 2021, Brussels, Belgium. pp.499-502, 10.1109/IGARSS47720.2021.9554273 . hal-03401587v1

\section{HAL Id: hal-03401587 \\ https://hal.science/hal-03401587v1}

Submitted on 25 Oct 2021 (v1), last revised 8 Nov 2021 (v2)

HAL is a multi-disciplinary open access archive for the deposit and dissemination of scientific research documents, whether they are published or not. The documents may come from teaching and research institutions in France or abroad, or from public or private research centers.
L'archive ouverte pluridisciplinaire HAL, est destinée au dépôt et à la diffusion de documents scientifiques de niveau recherche, publiés ou non, émanant des établissements d'enseignement et de recherche français ou étrangers, des laboratoires publics ou privés. 


\title{
POLARIMETRIC ANALYSIS USING THE ALGEBRAIC REAL REPRESENTATION OF THE SCATTERING MATRIX
}

\author{
Madalina Ciuca ${ }^{\star} \quad$ Gabriel Vasile Michel Gay $^{\star} \quad$ Andrei Anghel ${ }^{\S} \quad$ Silviu Ciochina ${ }^{\S}$ \\ * Grenoble Image Parole Signal et Automatique (GIPSA-lab), Saint-Martin-d'Hères, France \\ $\S$ University POLITEHNICA of Bucharest, Bucharest, Romania
}

\begin{abstract}
Equivalent matrix representations in radar polarimetry have long been studied and used as tools for modeling and understanding the scattering mechanisms. We include here the Kennaugh, Graves, or covariance matrices which are today seen as alternative representations of the same physical quantity, the scattering matrix.

In this paper, we briefly explore some of the properties of the algebraic real representation of a complex matrix, a mathematical construction which has been introduced in the literature as an alternative way of performing consimilarity transformations (rather than by the usual Takagi decomposition of a symmetric power matrix).

Besides the theoretical presentation on the subject, the main goal of the paper is to study some of the advantages and limitations of using the $4 \times 4$ real matrix form for representing the $2 \times 2$ complex scattering matrix.
\end{abstract}

Index Terms - Polarimetry, real matrix representation, consimilarity, similarity, SAR, coneigenvectors, coneigenvalues.

\section{INTRODUCTION}

The current paper proposes an investigation on the use of an alternative method to represent the scattering matrix of radar polarimetry and from there, to eventually retrieve new information associated to the backscattering process.

The rest of the document is structured as follows: Section 2 will provide a basic overview of concepts from linear algebra which are of primary importance for the current investigation, Section 3 will focus on implementation details and Section 4 will offer a brief conclusion.

Throughout the paper, the following notations will be used: bold characters with uppercase/lowercase format are utilized for matrices/vectors (e.g., A/b), while the notations for complex conjugate and transpose will be, as usual: $(\cdot)^{*},(\cdot)^{T}$. Moreover, $\operatorname{Re}(\cdot)$ and $\operatorname{Im}(\cdot)$ are used as operators for selecting the real and imaginary part of a given quantity.

\section{THE REAL REPRESENTATION OF A COMPLEX MATRIX}

\subsection{General definition}

Consider a generic square matrix having complex elements, $\mathbf{A} \in \mathbb{C}^{\mathbf{n} \times \mathbf{n}}$. We can find in the literature two definitions for its real representation [1]: a so-called, block-skew-circulant form (eq. 1) and a block-Hankel-skew-circulant form (eq. 2):

$$
\begin{aligned}
& \mathbf{A}_{\mathbf{R R}_{\mathbf{1}}}=\left[\begin{array}{cc}
\operatorname{Re}(\mathbf{A}) & -\operatorname{Im}(\mathbf{A}) \\
\operatorname{Im}(\mathbf{A}) & \operatorname{Re}(\mathbf{A})
\end{array}\right] \\
& \mathbf{A}_{\mathbf{R R}_{\mathbf{2}}}=\left[\begin{array}{cc}
\operatorname{Re}(\mathbf{A}) & \operatorname{Im}(\mathbf{A}) \\
\operatorname{Im}(\mathbf{A}) & -\operatorname{Re}(\mathbf{A})
\end{array}\right]
\end{aligned}
$$

In [2], Ling and Jiang have been the first to propose using the form from eq. 2 in the domain of radar polarimetry, while other resources have also supported the use of such a transformation for the case when a complex conjugation operator appears [1],[3]. As a result, this representation will also be used in the current article and hereafter referred generically as the real representation (RR) of a complex matrix. We will then investigate what information can be extracted when writing the scattering matrix - $\mathbf{S}$ - in this equivalent form, where it becomes a $4 \times 4$ matrix with only real elements, $\mathbf{S}_{\mathrm{RR}}$.

\subsection{Change of basis}

The methodology of basis change has been linked in radar polarimetry, for a long time, to that of finding the optimal polarization for a given scattering matrix (or, finding the states for which the radar receives minim/maximum power on certain directions). Such an analysis is also used in the case of other polarization matrices, for example the Jones matrix. Moreover, the scattering and Jones matrices may be connected themselves by a basis transformation [4], which appears as the result of using two different conventions for describing the scattering phenomenon on the receiving path.

A particular case of basis transformations are the ones which bring the matrix of interest to a diagonal form. Transforming the Jones matrix to another basis requires the use of a similarity transformation (eq. 3), operation which can be quite straightforward for most cases, even for the diagonalization (i.e., equivalent to an eigen-analysis). However, 
when performing a basis transformation of the scattering matrix, one must take into account, this time, a consimilarity (i.e., conjugate similarity) transformation (as it is described through the use of matrix $\mathbf{X}$, in eq. 4 ):

$$
\begin{gathered}
\mathbf{D}_{\mathbf{J}}=\mathbf{Y}^{-\mathbf{1}} \mathbf{J Y} \\
\mathbf{D}_{\mathbf{S}}=\left(\mathbf{X}^{*}\right)^{-\mathbf{1}} \mathbf{S X}
\end{gathered}
$$

Using the similarity approach and the Jordan canonical form(s), [5] classifies the Jones matrices based on the relationship between their eigenvalues and eigenvectors, as: diagonalizable homogeneous (i.e., with orthogonal eigenvectors) and inhomogeneous (i.e., only independent eigenvectors), as well as defective matrices (the latter are not diagonalizable, but can be reduced to an upper triangular Jordan canonical form).

More earlier, Lüneburg has performed a similar investigation [6], [7] taking also into account the case of the scattering matrix and formulating some observations for its coneigenvectors and coneigenvalues. A coneigenvector is "a polarization vector that does not change its state of polarization in case of backscattering".

$$
\mathbf{S x}=\xi \mathbf{x}^{*}
$$

In eq. 5 , we identify $\mathbf{x}$ as the coneigenvector and $\xi$ as its associated coneigenvalue. These quantitites are computed in the literature based on either simplifying assumptions (e.g., $\mathbf{S}$ is symmetric) or by using indirect methods: equivalent representations where the consimilarity transformation becomes a simple similarity one. For the first case, it has been reported that "every symmetric matrix is unitary congruent to a diagonal matrix with real nonnengative entries". In the second case, we include the method based on the power (Graves) matrix [6], as well as the one using the algebraic real representation of a complex matrix [2]. The brief schematic from Fig. 1 shows that by transferring the scattering matrix to a proper equivalent form, a similarity transformation can be used instead of the conjugate-similarity one.

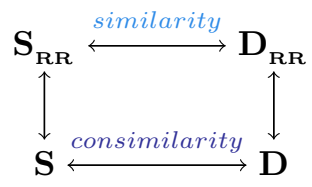

Fig. 1: Transformation equivalences in/between the space of the scattering matrix (bottom) and the space of the its real representation (top).

While the earlier approaches in radar polarimetry have used the equivalent representation with the power matrix, we are going to explore the more recent method with the real representation.

[8] and [2] show that one link exists between the eigenvalues of the RR and the coneigenvalues of the matrix from the original space. In the RR space, each real eigenvalue will be part of a pair $\left(\lambda_{k},-\lambda_{k}\right)$, in which both its positive and negative values are present. According to [2], the value $\lambda_{k}$ corresponds to the modulus of the coneigenvalue $\xi_{k}, k \in$ $\{1,2\}$. A lost of information referring to the original phase term may appear if in the original space the coneigenvalue was complex. The same situation also exists in case of using the Graves method.

We recall that when a square matrix with real elements presents a complex eigenvalue, also its complex conjugate will appear as eigenvalue. This general property adds to the one presented earlier and, as a result, if an eigenvalue of the RR matrix is complex, it will be part of a quad set: $\left(\lambda_{k},-\lambda_{k}, \lambda_{k}^{*},-\lambda_{k}^{*}\right)$.

With this in mind, and because $\mathbf{S}_{\mathbf{R R}} \in \mathbb{R}^{4 \times 4}$ we can identify two main combinations: $\mathbf{S}_{\mathbf{R R}}$ presents either only real eigenvalues (two distinct pairs or one double pair) or only complex ones. Each pair of real eigenvalues relates to a coneigenvalue, while each quad set of complex eigenvalues corresponds to only one. Each coneigenvalue, $\xi_{k}$, will have associated one coneigenvector, $\mathbf{x}_{\mathbf{k}}$.

In the space of the real representation, the eigenvector associated to the negative eigenvalue, $-\lambda_{k}$, can be obtained through a simple relationship from the one which corresponds to eigenvalue $\lambda_{k}$ :

$$
\mathbf{S}_{\mathbf{R R}} \mathbf{y}_{\mathbf{k}}=\lambda_{k} \mathbf{y}_{\mathbf{k}} \quad \mathbf{S}_{\mathbf{R R}}\left(\mathbf{Q}_{\mathbf{2}} \mathbf{y}_{\mathbf{k}}\right)=-\lambda_{k}\left(\mathbf{Q}_{\mathbf{2}} \mathbf{y}_{\mathbf{k}}\right)
$$

where

$$
\mathbf{Q}_{\mathbf{2}}=\left(\begin{array}{cc}
\mathbf{0} & -\mathbf{I}_{\mathbf{2}} \\
\mathbf{I}_{\mathbf{2}} & \mathbf{0}
\end{array}\right)=\left(\begin{array}{cccc}
0 & 0 & -1 & 0 \\
0 & 0 & 0 & -1 \\
1 & 0 & 0 & 0 \\
0 & 1 & 0 & 0
\end{array}\right)
$$

If $\lambda_{k}$ would be a complex eigenvalue, we already know that the eigenvector associated to $\lambda_{k}^{*}$ is itself a conjugated version of $\mathbf{y}_{\mathbf{k}}$ :

$$
\mathbf{S}_{\mathbf{R R}}^{*} \mathbf{y}_{\mathbf{k}}^{*}=\lambda_{k}^{*} \mathbf{y}_{\mathbf{k}}^{*} \rightarrow \mathbf{S}_{\mathbf{R R}} \mathbf{y}_{\mathbf{k}}^{*}=\lambda_{k}^{*} \mathbf{y}_{\mathbf{k}}^{*}
$$

Based on this observations, it is easier to understand why the case of a quad set in the RR space returns only one coneigenvalue/coneigenvector pair.

By investigating its real space representation, we can link a general interpretation for the scattering matrix, $\mathbf{S}$, which can be:

- con-diagonalizable homogeneous: when $\mathrm{S}_{\mathrm{RR}}$ has real and distinct eigenvalues with orthogonal eigenvectors.

- con-diagonalizable inhomogeneous: when $\mathbf{S}_{\mathrm{RR}}$ has real eigenvalues and independent eigenvectors.

- con-defective: when $\mathrm{S}_{\mathrm{RR}}$ has complex eigenvalues, which will result in one complex and one zero coneigenvalue. 


\section{IMPLEMENTATION}

In the following, we intend to use the space of the RR for performing a polarimetric analysis.

\subsection{Parametric representation}

In the algebraic space of the real representation, each real matrix $S_{R R}$ has at least one eigenvalue with one unique eigenvector (represented as a $4 \times 1$ column vector). We can interpret each line entry from the vector as coordinates spanning a 4D space. Because they have normalized values, all possible positions where the tip of the vector can be located are contained inside or on the surface of a unit $S^{3}$ sphere (or, 3-sphere).

In radar polarimetry, a very common method is to perform the representation of some scattering parameters (e.g., the Stokes vectors) on a $S^{2}$ (3D) sphere, known as the Poincaré sphere. Generally, in a spherical coordinate system, we can write the position of a point by specifying 3 parameters, a radius (r) and two angles $(\theta, \phi)$ :

$[\mathrm{r} \sin \theta \cos \phi, r \sin \theta \sin \phi, r \cos \theta]^{T}$.

However, the convention used for writing the Stokes parameters is different in terms of the latitude angle and always considers a radius equal to one: $[\cos \beta \cos \phi, \cos \beta \sin \phi, \sin \beta]^{T}$. Thus, the $\beta$ angle is the complementary of $\theta$ :

$$
\beta=\pi / 2-\theta .
$$

We now introduce the definition of the Hopf map, a manyto-one continuous function from the $S^{3}$ sphere to the $S^{2}$ sphere, such that each distinct great circle from the 3-sphere maps to a single point on the 2-sphere [9], [10].

[11] writes the Hopf coordinates on the 3-sphere, by means of some angular values from the 2-sphere, as:

$$
\begin{aligned}
& {[\cos (\theta / 2) \cos (\phi+\Phi), \cos (\theta / 2) \sin (\phi+\Phi),} \\
& \sin (\theta / 2) \cos \Phi, \sin (\theta / 2) \sin \Phi]^{T}
\end{aligned}
$$

where $\phi, \Phi \in[0,2 \pi]$ and $\theta \in[0, \pi]$.

Mapping each element of the vector in eq. 9 to a corresponding element from an eigenvector in the real representation space,

$\mathbf{y}_{\mathbf{k}}=[y(1, k), y(2, k), y(3, k), y(4, k)]^{T}$, we can extract the angular values on the 3 -sphere:

$$
\begin{aligned}
\Phi & =\arctan \left(\frac{y(4, k)}{y(3, k)}\right) \\
\phi & =\arctan \left(\frac{y(2, k)}{y(1, k)}\right)-\Phi \\
\theta & =2 \arctan \left(\frac{y(3, k)}{y(1, k)} \cdot \frac{\phi}{\Phi}\right)
\end{aligned}
$$

and then, use the angular values $(\beta, \phi)$ for performing the mapping on the 2 -sphere.

\subsection{Results and discussion}

We use the previously introduced parametric representation on the 2-sphere and investigate the distribution in fullpolarimetric radar images, for scatterers in distinctive zones, chosen to be characterized by different scattering mechanisms.

We start the investigation with a monostatic X-Band ONERA RAMSES full-pol data set [12]. Three zones of interest are identified in the image and $20 \times 20$ pixels selection boxes (red, green and blue) are used (Fig. 2a).

For the three zones, we extract at the level of each pixel the largest eigenvalue and the associated eigenvector. After determining the projective angles $(\beta, \phi)$, as presented in subsection 3.1, the eigenvalue modulus is taken as the length of the radius from the center of the sphere. If $\left|\lambda_{1}\right| \geq 1$, the whole set of eigenvalues is scaled so that $\left|\lambda_{1}\right|=1$ and we can map it maximum on the surface of the sphere.

Different behaviours can be observed for the pixels from each zone: the highly reflective scatterers from the red zone are mainly represented on the surface of the sphere and have a tendency of accumulating at/around its North and South poles. The pixels from the blue box are representative of the volumetric scattering type and they appear to have random positions, with the great majority of them lying inside the sphere. For the green area, the pixels representation seems to be a combination of the two previous ones, with points lying both inside the sphere as well as on its surface.

\section{CONCLUSIONS}

In the present article we have examined some of the general properties of the RR of a complex matrix, with a particular focus on the RR of the radar scattering matrix. Also, a parametric representation of the eigenvectors from the RR space has been proposed and used for displaying the dominant eigen-components from zones described by different scattering mechanisms.

It is intended that more results with simulated (and/or real) data sets will be included in the final version paper, while respecting the remained space limit.

\section{REFERENCES}

[1] C. Hellings and W. Utschick, "Two different real representations of complex matrices for describing widely linear systems," in Proceedings of the International Symposium on Wireless Communication Systems, Aug. 2015, pp. 641-645.

[2] S. Ling and T. Jiang, "New method for general Kennaugh's pseudo-eigenvalue equation in radar polarimetry," Frontiers of Mathematics in China, vol. 7, no. 1, pp. 85-95, 2012. 


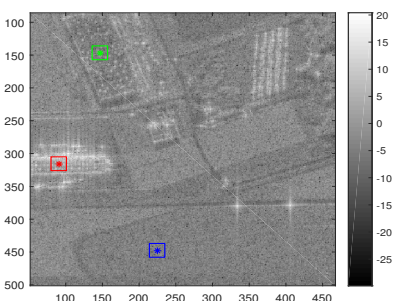

(a) Bretigny X-Band PolSAR HH channel

(red, green, blue: regions of interest)

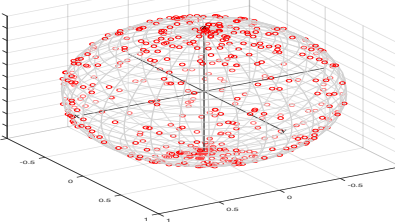

(b) Red box: highly reflective scatterers (1st eigenvector)

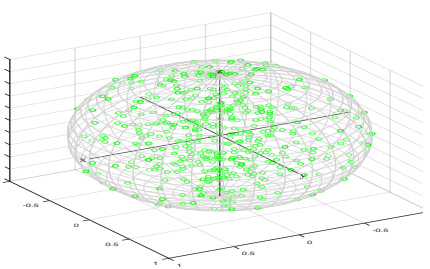

(c) Green box (1st eigenvector)

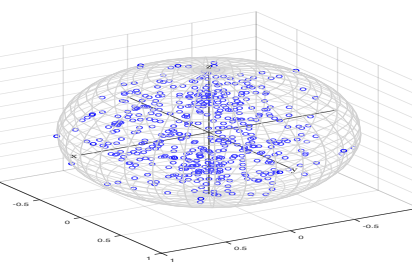

(d) Blue box: volumetric scatterers (1st eigenvector)

Fig. 2: Selection of scatterers parametric representation.

[3] T. Jiang and M. Wei, "On the reduction of a complex matrix to triangular or diagonal by consimilarity," $\mathrm{Nu}$ merical Mathematics-English Series-, vol. 15, no. 2, pp. 107-112, 2006.

[4] T. Dallmann and D. Heberling, "On the connection between Jones matrix and Sinclair matrix," in Progress In Electromagnetics Research Symposium (PIERS), 2015, pp. 258-262.

[5] J.C. Gutiérrez-Vega, "The field of values of Jones matrices: classification and special cases," Proceedings of the Royal Society A, vol. 476, no. 2242, 2020.

[6] E. Lüneburg and WM. Boerner, "Consimilarity classification of general radar scattering matrices," in Proceedings of the International Symposium on Antennas and Propagation, 1996, vol. 4, pp. 1041-1044.

[7] E. Lüneburg and WM. Boerner, "Homogeneous and inhomogeneous Sinclair and Jones matrices," in Wideband Interferometric Sensing and Imaging Polarimetry. International Society for Optics and Photonics, 1997, vol. 3120, pp. $45-54$.

[8] T. Jiang, X. Cheng, and L. Chen, "An algebraic relation between consimilarity and similarity of complex matrices and its applications," Journal of Physics A: Mathematical and General, vol. 39, no. 29, pp. 9215-9222, 2006.

[9] F. A. Rodrigues, G. Temporão, and J. P. von der Weid, "Discrete Hopf fibration in the design of fourdimensional modulations," in 2017 SBMO/IEEE MTT$S$ International Microwave and Optoelectronics Conference (IMOC), 2017, pp. 1-5.

[10] F. Rodrigues, G. Temporão, and J P von der Weid, "Constructive methods for the design and labeling of fourdimensional modulations," Journal of Communication and Information Systems, vol. 33, no. 1, Aug. 2018.

[11] M. Zamboj, "Synthetic construction of the Hopf fibration in the double orthogonal projection of the 4-space," arXiv preprint arXiv:2003.09236, 2020.

[12] G. Vasile and L. Pralon, "Blind source separation in polarimetric SAR interferometry," in IEEE International Geoscience and Remote Sensing Symposium, 2016, pp.
$5650-5653$. 